\title{
Correction of Motional Electric Field Measurements for Galvanic Distortion*
}

\author{
Alan D. Chave \\ Deep Submergence Laboratory, Department of Applied Ocean Physics and Engineering, Woods Hole Oceanographic Institution, \\ Woods Hole, Massachusetts \\ DOUGLAS S. LUTHER \\ Department of Oceanography, University of Hawaii at Manoa, Honolulu, Hawaii \\ CHRistopher S. MeInen \\ Cooperative Institute for Marine and Atmospheric Studies, University of Miami, Miami, Florida
}

(Manuscript received 30 April 2002, in final form 3 August 2003)

ABSTRACT

\begin{abstract}
Interactions between motional electric fields and lateral gradients in electrical conductivity (e.g., seafloor topography) produce boundary electric charges and galvanic (i.e., noninductive) secondary electric fields that result in frequency-independent changes in the electric field direction and amplitude that are specific to a single location. In this paper, the theory of galvanic distortion of the motional electric field is developed from first principles and a procedure to correct for it is then derived. The algorithm is based on estimation of intersite transfer tensors for the horizontal electric fields at the high frequencies where external (ionospheric and magnetospheric) sources, not oceanic motionally induced electric fields, dominate. A decomposition of each measured tensor is derived that expresses it as the product of a set of distortion tensors and the underlying, undistorted transfer tensor. The algorithm may be applied simultaneously to a set of sites and assessed statistically, yielding the undistorted electric field uniquely at each site except for a single site-dependent multiplicative scalar, which must be obtained from other data. Because the distortion is frequency independent, the same tensors may be used to undistort the low-frequency, motional induction components that are of interest in oceanography. This procedure is illustrated using an electric field dataset collected in the Southern Ocean in 1995-97, which is significantly distorted by galvanic processes.
\end{abstract}

\section{Introduction}

Electric fields are induced in the ocean by external, ionospheric and magnetospheric, electric current systems, and by the flow of ocean water currents through Earth's magnetic field. The latter, motional component of the electric field, is of oceanographic interest because it is a natural vertical average of the horizontal water velocity. Integrating variables like the electric field isolate physical processes that are difficult to examine using point (e.g., current meter) measurements, and can yield more accurate estimates of climatically important

* Woods Hole Oceanographic Institution Contribution Number 10977.

Corresponding author address: Dr. Alan D. Chave, Deep Submergence Laboratory, Dept. of Applied Ocean Physics and Engineering, Woods Hole Oceanographic Institution, MS 7, Woods Hole, MA 02543.

E-mail: alan@whoi.edu quantities like volume or heat transport. See Luther and Chave (1993) for elaboration.

The theory of motional induction in the low-frequency (subinertial) limit has been most comprehensively developed by Sanford (1971), Chave and Luther (1990), and Larsen (1992). For a flat-bottomed ocean at low frequencies, when the aspect ratio of ocean currents is small, when the effect of self-induction is weak, when the vertical velocity can be neglected in comparison to the horizontal components, and ignoring any noise contribution from external sources, it can be shown that the horizontal electric field (HEF) at a point is given by

$$
\mathbf{E}_{h}^{o}=\Lambda F_{z} \hat{z} \times\left\langle\mathbf{v}_{h}\right\rangle *+\mathbf{J} * / \sigma_{o} .
$$

The first term in (1) is a local (i.e., at the measurement point) average of the water velocity and is polarized in planes containing the vertical. The quantity $\left\langle\mathbf{v}_{h}\right\rangle *$ is the seawater conductivity weighted, vertically integrated (CWVI) horizontal water velocity, 


$$
\left\langle\mathbf{v}_{h}\right\rangle^{*}=\frac{\int_{-H}^{0} \sigma_{o}(z) \mathbf{v}_{h}(z) d z}{\int_{-H}^{0} \sigma_{o}(z) d z},
$$

averaged horizontally over a radius of order the water depth centered on the measurement point (Chave and Luther 1990), where $F_{z}$ is earth's vertical magnetic induction, $\Lambda$ is a scale factor that depends primarily on the leakage of electric current into the seafloor, and $\sigma_{o}$ is the depth-dependent electrical conductivity of seawater.

The second term $\mathbf{J} * / \sigma_{o}$ in (1) represents nonlocally generated electric currents that flow in the horizontal plane. These may be produced by downstream changes (e.g., meandering) of narrow currents, by topography, and by distant fluid motion, among other causes (Sanford 1971; Larsen 1992). Since $\mathbf{J} * / \sigma_{o}$ appears additively in (1) and reflects the water velocity at various points in a region surrounding a measurement, the nonlocal current term can obscure the oceanographically interesting CWVI velocity if it is sufficiently large. This is quite distinct from the effect of changes in seafloor conductance contained in $\Lambda$, which appear effectively as calibration changes on the CWVI velocity.

In the absence of independent measurements of nonlocal electric currents, (1) is typically rearranged to yield

$$
\left\langle\mathbf{v}_{h}\right\rangle^{*}=\frac{\mathbf{E}_{h}^{o}}{\Lambda F_{z}} \times \hat{z} .
$$

It must be recognized that nonlocal electric currents will appear as additive noise to the electric field in (3). This is most effectively detected by comparing independent electric field and conventional estimates of the CWVI velocity.

Numerous comparisons of electric field observations taken over a wide range of flow regimes with independent estimates of the CWVI velocity have demonstrated that the nonlocal current term in (1) is small [typically $1 \mathrm{~cm} \mathrm{~s}^{-1}$ or less; e.g., Cox (1980), Sanford (1986), Spain and Sanford (1987), Luther et al. (1991), Larsen (1992), Luther and Chave (1993), and Sanford et al. (1995)]. Numerical models of motional induction incorporating real topography further support this assertion. Flosadottir et al. (1997a,b) derived motional electric fields in the presence of real topography from the the World Ocean Circulation Experiment (WOCE) Community Modelling Effort (CME) for the North Atlantic, showing that nonlocal current effects are negligible over most of the basin. Tyler et al. (1997a,b) also suggest that nonlocal current effects are second order compared to the uncertainty in estimating $\Lambda$ over most of the world's oceans. While all of these results suggest that the second term in (1) can be neglected compared to the first, that conclusion will be further examined in section 6 .

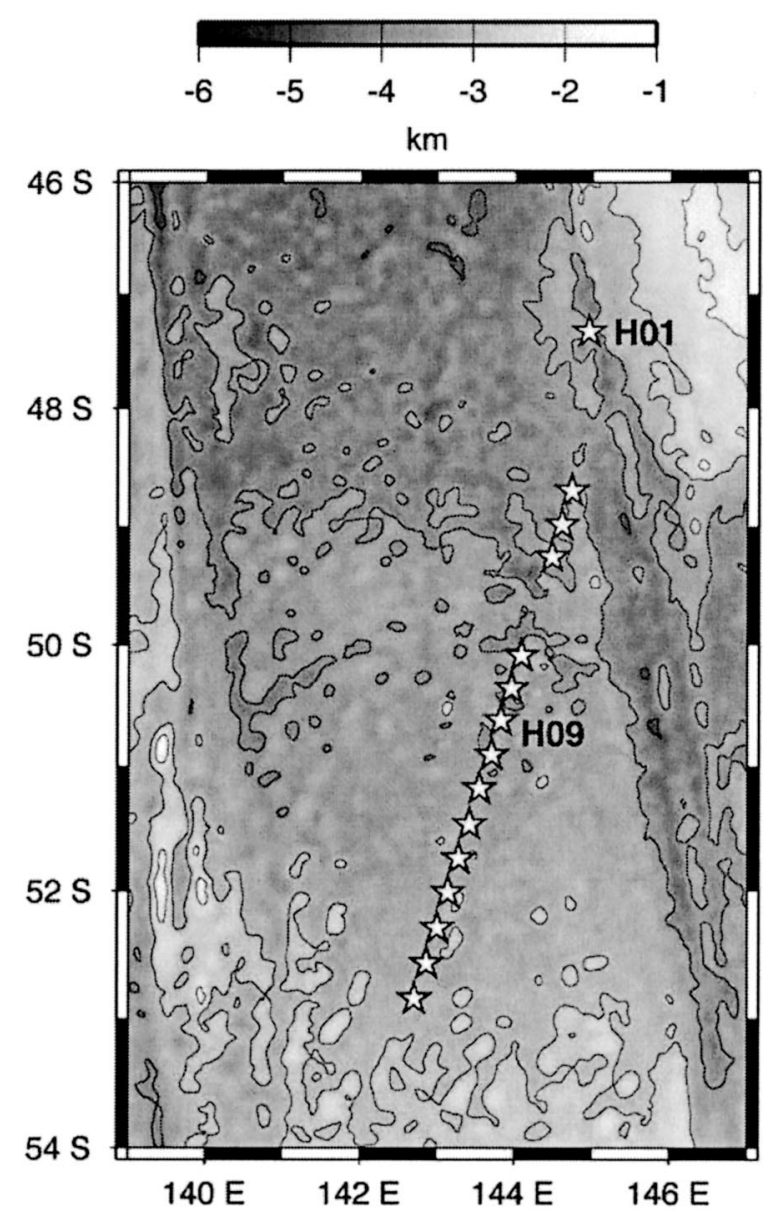

FIG. 1. Bathymetric map showing the location of the SAFDE horizontal electric field recorders (white stars). H01 is at the north end and $\mathrm{H} 17$ is at the south end (see Table 1). The gap at about $49.5^{\circ} \mathrm{S}$ is due to lost instruments $\mathrm{H} 05$ and H06. The HEF array is bounded immediately $(\sim 30 \mathrm{~km})$ to the east and west by inverted echo sounders over the middle $2 / 3$ of the line, and an array of nine current meter moorings was located near H09. Bathymetry is from Smith and Sandwell (1997).

\section{The SAFDE dataset}

During the recent Sub-Antarctic Flux and Dynamics Experiment (SAFDE; Luther et al. 1997) located southwest of Tasmania, a 17-element, 400-km-long linear array of horizontal electrometers was placed on the rugged, nearly sediment-free north flank of the Southeast Indian Ridge (Fig. 1). SAFDE was designed to obtain observations of the Antarctic Circumpolar Current and permit direct evaluation of the momentum, energy, and vorticity budgets of the most poorly characterized ocean current on Earth. The main part of the SAFDE array was in the water from April 1995 through March 1997. The instrumentation consisted of a central $70-\mathrm{km}$-diameter array of nine subsurface current meter moorings and a linear array of 17 horizontal electrometers and 18 inverted echo sounders extending SSW-NNE and overlapping the WOCE SR3 hydrographic line. 
TABLE 1. SAFDE horizontal electrometer deployments.

\begin{tabular}{lcccc}
\hline \hline Site & S lat & E lon & Depth $(\mathrm{m})$ & $\begin{array}{c}\text { Duration } \\
\text { (days) }\end{array}$ \\
\hline H01 & $47^{\circ} 19.83^{\prime}$ & $144^{\circ} 57.55^{\prime}$ & 4044 & $702^{*}$ \\
H02 & $48^{\circ} 41.93^{\prime}$ & $144^{\circ} 44.30^{\prime}$ & 4018 & 706 \\
H03 & $48^{\circ} 59.24^{\prime}$ & $144^{\circ} 36.30^{\prime}$ & 3929 & 705 \\
H04 & $49^{\circ} 15.76^{\prime}$ & $144^{\circ} 28.69^{\prime}$ & 4187 & 707 \\
H07 & $50^{\circ} 05.12^{\prime}$ & $144^{\circ} 05.28^{\prime}$ & 3696 & 710 \\
H08 & $50^{\circ} 21.43^{\prime}$ & $143^{\circ} 57.42^{\prime}$ & 4166 & 716 \\
H09 & $50^{\circ} 37.49^{\prime}$ & $143^{\circ} 49.10^{\prime}$ & 3701 & 726 \\
H10 & $50^{\circ} 54.19^{\prime}$ & $143^{\circ} 42.14^{\prime}$ & 4268 & 726 \\
H11 & $51^{\circ} 10.83^{\prime}$ & $143^{\circ} 32.47^{\prime}$ & 3758 & 722 \\
H12 & $51^{\circ} 28.19^{\prime}$ & $143^{\circ} 25.04^{\prime}$ & 3661 & 208 \\
H13 & $51^{\circ} 44.66^{\prime}$ & $143^{\circ} 16.67^{\prime}$ & 3576 & 724 \\
H14 & $52^{\circ} 00.56^{\prime}$ & $143^{\circ} 08.19^{\prime}$ & 3667 & 724 \\
H15 & $52^{\circ} 17.24^{\prime}$ & $142^{\circ} 59.72^{\prime}$ & 3492 & 92 \\
H16 & $52^{\circ} 34.10^{\prime}$ & $142^{\circ} 51.65^{\prime}$ & 3502 & 726 \\
H17 & $52^{\circ} 50.46^{\prime}$ & $142^{\circ} 42.02^{\prime}$ & 2986 & 547 \\
\hline
\end{tabular}

* Single component.

The operating principles for the horizontal electrometer are discussed by Filloux (1987), Petitt et al. (1992), and Bailey et al. (2001) and include the use of mechanical water switching technology to eliminate lowfrequency electrode drift. Of the 17 electrometers deployed, 15 were recovered, yielding 11 full and 4 partial records (Fig. 1; Table 1). The measured electric fields were converted to initial estimates of the CWVI velocity using (3) by assuming $\Lambda=1$ and taking the vertical geomagnetic field from the International Geomagnetic Reference Field 1995 (Macmillan et al. 1997; Quinn et al. 1997) corrected for secular variation to the middle of the SAFDE epoch.

In the course of instrument debriefing, systematic and substantial (up to a factor of 2) intersite amplitude variations were detected that cannot be explained instrumentally. All instruments were calibrated pre- and postdeployment, indicating no measurable change in the system transfer functions from electrode inputs through digital recording. Off-channel leakage in the water switches can reduce the on-channel signal amplitude (Filloux 1987), but it was systematically measured at recovery and never exceeded a few percent.

Further investigation shows that the amplitude shifts are systematic, similarly influencing both the short $(<1$ day) period range that is controlled by external, ionospheric sources and the long period range ( $>3$ day) that is dominated by oceanic motional induction. Figure 2a compares the standard deviation of the current speed estimates for the 12 most complete records (eliminating H01, H12, and H15) after low-pass filtering (72-h cutoff) to isolate motional induction and bandpass filtering $(3.8-2.5 \mathrm{~h})$ to isolate a part of the spectrum that is dominated by auroral activity but not influenced by the ocean and ionospheric tides. In each case, a four-pole Butterworth filter was applied forward and backward over the data series. Note that the site-to-site amplitude shifts are very similar in the long and short period bands. A general quasi-linear increase to the south in the short period
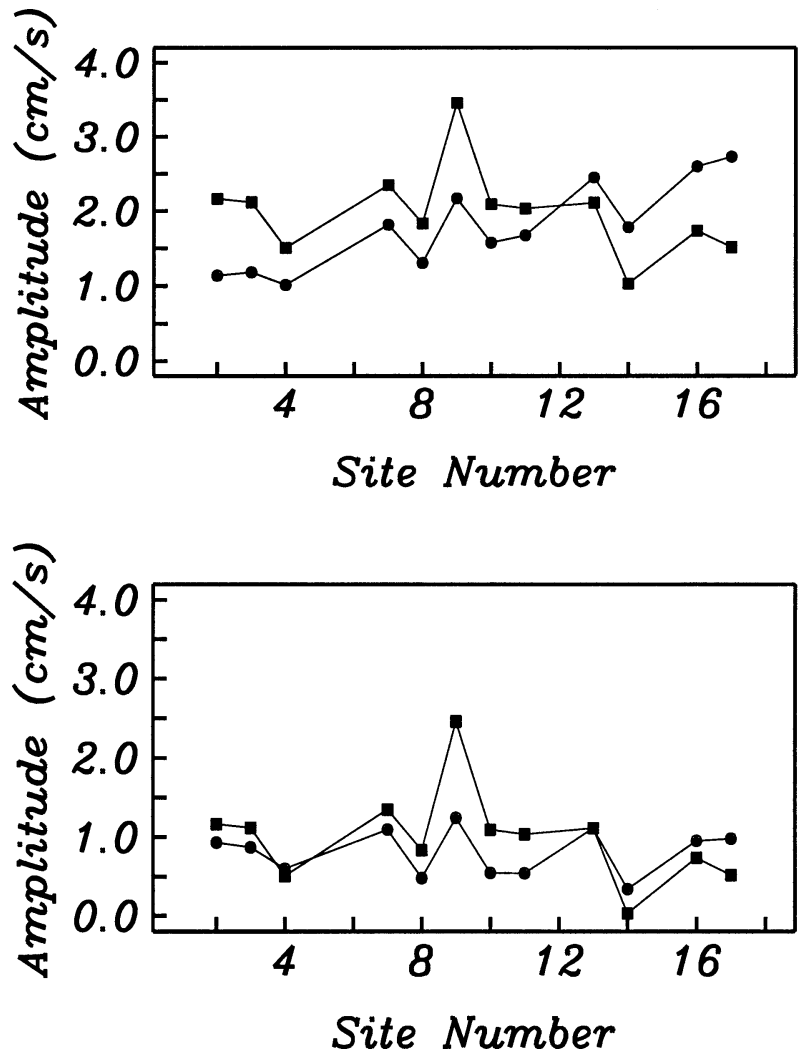

FIG. 2. Std dev of the magnitude of the time series after bandpass filtering over $3.8-2.5 \mathrm{~h}$ (solid circles) to isolate the auroral substorm band and lowpass filtering at $72 \mathrm{~h}$ (solid squares) to isolate the motional induction band. The site variance for the motional induction band has been scaled to equal that in the auroral substorm band, resulting in a reduction of amplitude of about 0.5 , and the motional induction band data have been shifted down by (top) 1 and (bottom) 2 for clarity. The top panel shows the amplitude data without correcting the auroral substorm data for a general amplitude increase toward the geomagnetic pole. The bottom panel shows these data after removal of a linear trend.

band is easily explained by increasing proximity to the overhead auroral currents as the geomagnetic pole is approached (see section 6 for details). Figure $2 \mathrm{~b}$ shows the data from Fig. 2a after removing a linear trend from the short period data, which makes the correlation of short and long period amplitudes even more striking.

Motional electric fields at long periods are influenced both by nonlocal electric currents and, since they are polarized in the vertical plane, by changes in the vertical conductance beneath the seafloor [parameterized by $\Lambda$ in (1) and (3)]. Because of the very low electrical conductivity in Earth's mantle (e.g., Chave et al. 1990), changes in $\Lambda$ are usually dominated by fluctuations in the thickness of relatively conductive sediments, as further discussed by Chave and Luther (1990) and Tyler et al. (1997b). By contrast, because the atmosphere is nearly insulating, electric fields induced by external sources are polarized in the horizontal plane and couple to Earth principally by induction. Using skin depth ar- 
guments, as well as more formal modeling, it is easy to show that the externally induced electric field is quite insensitive to the surface electrical structure (especially the sedimentary layer) at a period of $\sim 3 \mathrm{~h}$. In fact, it is most sensitive to structure below $100-\mathrm{km}$ depth. In addition, the long period nonlocal electric currents have no analog in the short period band. The similarity of the short and long period amplitude variations in Fig. 2 strongly suggests a common causative process for the amplitude fluctuations, but this cannot be nonlocal electric currents because they do not exist at periods dominated by external sources. Similarly, sediment thickness variations have little effect at short periods, and cannot alone explain the observations in Fig. 2. In any case, the SAFDE region is nearly devoid of sediment (Hayes and LaBrecque 1991). This suggests that some other process that was not fully considered in deriving (1)(3) is responsible.

\section{Galvanic distortion of the electromagnetic field}

Both (1)-(3) and the previous arguments are essentially one-dimensional in that they neglect lateral variations in the electrical conductivity within the ocean and below the seafloor. Sanford (1971) did incorporate topography in his derivation of (1) through perturbation analysis and does account for lowest-order galvanic effects on the nonlocal current term, but of necessity the result is an incomplete description of a complicated phenomenon. In the real ocean, lateral conductivity changes occur over a continuum of scales ranging from meters (e.g., a boulder near a seafloor instrument) to tens of kilometers (e.g., a seamount) to basin scales (e.g., a continental margin), and may occur within the sediments and crust as well as at the seafloor. It is well known in electromagnetic geophysics (e.g., the magnetotelluric method) that boundary electric charges on any of these structures will distort regional electromagnetic fields. In fact, this phenomenon is pervasive, and correction for it is a routine part of magnetotelluric data analysis. In the galvanic limit that typically obtains, the distortion becomes frequency independent, consistent with the observations in Fig. 2.

Reviews of electromagnetic distortion appear in Jones (1983), Berdichevsky et al. (1989), Jiracek (1990), and Groom and Bahr (1992). The relevant physics is quite simple. Galvanic distortion is caused by the primary or regional electric field $\mathbf{E}_{p}$ producing electric charges at gradients in electrical conductivity (Fig. 3). While the charges themselves are minute (volume charge density $\rho_{v}=-\varepsilon \mathbf{E}_{p} \cdot \nabla \sigma / \sigma$, where $\varepsilon$ is the permittivity of the medium), the secondary electric field is given by Coulomb's law $\left(\mathbf{E}_{s}=1 /(4 \pi \varepsilon) \int \rho_{v} \mathbf{r} /|\mathbf{r}|^{2} d v\right)$ and may be quite large. This is especially true near the seafloor, where the conductivity contrast of rock with seawater or sediment is substantial. The accumulated charges result in secondary electric fields that add vectorially with the primary one, resulting in changes in both the di-
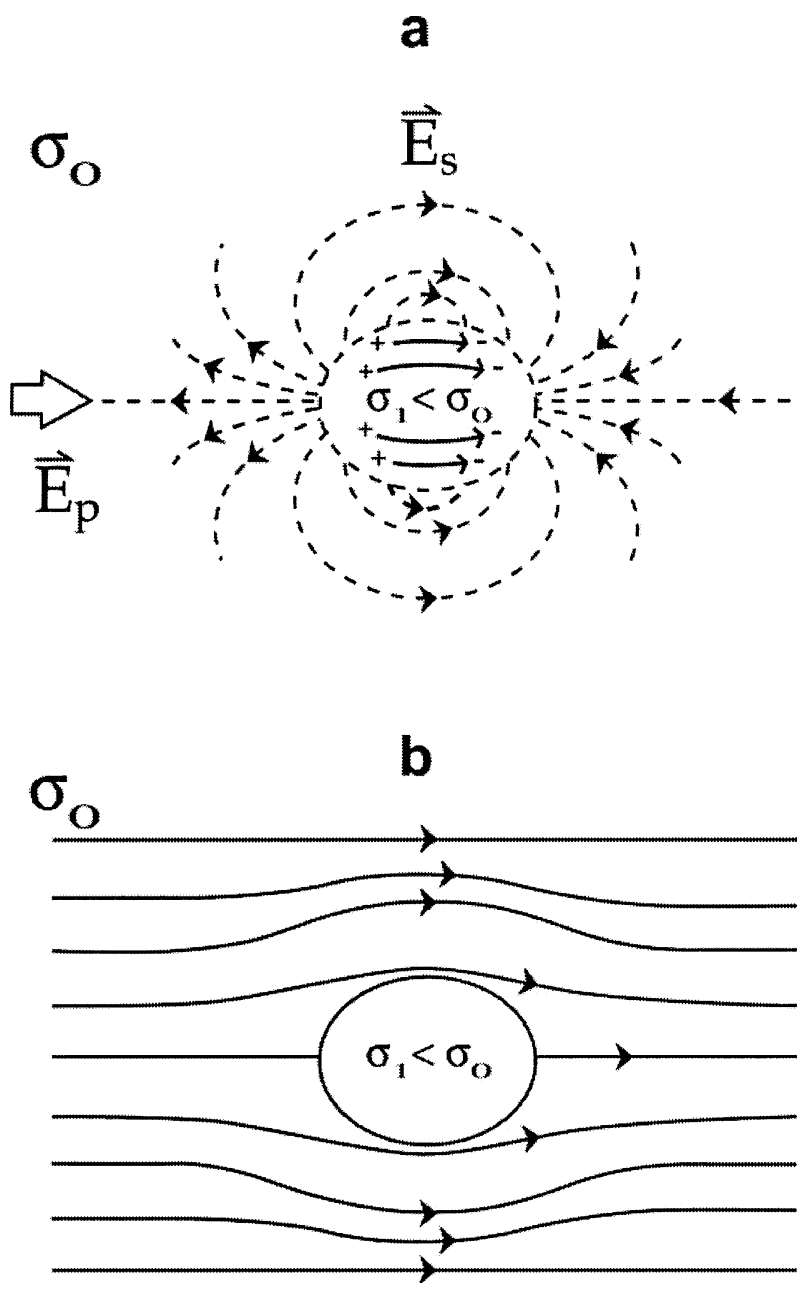

Fig. 3. Cartoon showing the galvanic distortion effect when the distorting body (conductivity $\sigma_{1}$ ) is more resistive than the background medium (conductivity $\sigma_{0}$ ), as would be typical at the seafloor. (a) A uniform primary electric field $\mathbf{E}_{p}$ directed to the right, which produces a boundary charge on the distorting body, which in turn produces a secondary electric field $\mathbf{E}_{s}$ (dashed lines). (b) The resulting distorted electric field, which is changed in both amplitude and direction, depending on location relative to the distorting body.

rection and amplitude of the observed electric fields and electric currents. In the galvanic limit, the primary and secondary electric fields are always in phase and hence the effect is frequency independent. For the nearly universal case of a resistive (relative to seawater) distorting body, the electric field will increase above the body and off the ends, but decrease along the sides (Fig. 3), and hence amplitude shifts may be upward or downward, as observed in Fig. 2. Directional changes in the electric field may also be significant, as demonstrated in section 6. Groom and Bailey (1991) give an analytical solution for the galvanic effect of a hemispherical distorting body in a background medium that further quantifies these points.

There are two main approaches to the removal of galvanic distortion from electromagnetic data: direct 
modeling and tensor decomposition. The former is rarely used because a priori information about the location, conductivity, and geometry of distorting bodies is typically inadequate. In the case of seafloor data, it might appear that multibeam bathymetry would suffice, but this presumes that the only important distortion is caused by the seawater-to-seafloor interface. Multibeam data do not provide information on the geometry or depth extent of sediment pockets or subsurface structures that may be as electrically important as topography. Nolasco et al. (1998) and Evans et al. (1999) did use a thin sheet approach to model large-scale seafloor distortion produced by regional bathymetry in the magnetotelluric limit. This was only partially successful because smaller-scale or buried distorters are unresolved. In addition, numerical modeling of distortion is complicated because a wide range of scales must be accurately simulated and because of strong coupling between the surface and deeper structures. For the SAFDE dataset, multibeam bathymetry is limited and regional information is sparse; hence, a direct modeling approach is not feasible even in the absence of numerical limitations.

In magnetotellurics, the fundamental datum is a frequency-domain tensor relating the observed electric and magnetic fields. Distortion removal based on a physicalmodel-derived mathematical decomposition of this tensor is now routinely employed, as described by Groom and Bailey (1989) and Chave and Smith (1994). This has the advantage that explicit information about the location and geometry of distorting bodies is not required, and can be assessed statistically.

\section{Theoretical description of galvanic distortion}

The full differential equation for the motional threecomponent electric field under the quasi-static (preMaxwell) approximation and with $e^{-i \omega t}$ time dependence suppressed is

$$
\begin{aligned}
\boldsymbol{\nabla} & \times \boldsymbol{\nabla} \times \mathbf{E}(\mathbf{r})-i \omega \mu_{o} \sigma_{o} \mathbf{E}(\mathbf{r}) \\
& =i \omega \mu_{o} \delta \sigma(\mathbf{r}) \mathbf{E}(\mathbf{r})+i \omega \mu_{o} \sigma(\mathbf{r})[\mathbf{v}(\mathbf{r}) \times \mathbf{F}],
\end{aligned}
$$

where $\mu_{o}$ is the permeability of free space, $\mathbf{F}$ is the static component of Earth's magnetic field, $\delta \sigma(\mathbf{r})=\sigma(\mathbf{r})-$ $\sigma_{o}$, and $\sigma_{o}$ represents the depth-dependent conductivity of a flat-bottomed ocean underlain by a horizontally layered, conductive earth. Departures of $\sigma(\mathbf{r})$ from $\sigma_{o}$ are principally due to seafloor topography and nonhorizontal structures within the sediments and crust, which scatter the induced electric field.

The background electric field $\mathbf{E}^{o}$ is the solution of (4) when $\delta \sigma(\mathbf{r})=0$ and hence $\sigma(\mathbf{r})=\sigma_{o}$. To lowest order, the horizontal components of $\mathbf{E}^{o}$ are given by (1). The vertical component is $\left(\mathbf{v}_{h} \times \mathbf{F}_{h}\right) \cdot \hat{z}$. Because of the local nature of the spatial averaging for the horizontal electric field and the strictly local relationship between the vertical electric field and the water velocity, $\sigma(\mathbf{r})$ will be approximated by $\sigma_{o}$ in the last term on the right-hand side of (4). This should be fairly accurate except in unusual situations where depth is changing very rapidly near the measurement point, such as at the edge of a large scarp or seamount.

Because the electric field at an observation point $\mathbf{r}$ may be in a different direction to that at a source point $\mathbf{r}^{\prime}$, the full solution of (4) must be obtained using a tensor or dyadic Green function, as described by Yaghian (1980) or Habashy et al. (1993). The dyadic Green function satisfies

$$
\boldsymbol{\nabla} \times \boldsymbol{\nabla} \times \mathbf{G}\left(\mathbf{r}, \mathbf{r}^{\prime}\right)-\gamma_{o} \mathbf{G}\left(\mathbf{r}, \mathbf{r}^{\prime}\right)=\mathbf{I} \delta\left(\mathbf{r}-\mathbf{r}^{\prime}\right),
$$

subject to appropriate boundary conditions, where $\gamma_{o}=$ $i \omega \mu_{o} \sigma_{o}$ and $\mathbf{I}$ is the identity tensor. For points external to the scattering body (which includes the seafloor), the electric field $\mathbf{E}$ is described by the integral equation

$$
\mathbf{E}(\mathbf{r})=\mathbf{E}^{o}(\mathbf{r})+\int_{V_{s}} d \mathbf{r}^{\prime} \mathbf{G}\left(\mathbf{r}, \mathbf{r}^{\prime}\right) \delta \gamma\left(\mathbf{r}^{\prime}\right) \mathbf{E}\left(\mathbf{r}^{\prime}\right),
$$

where $V_{s}$ is the volume of the distorting body. The integration domain may be taken over a volume extending from the seafloor to a point below which further conductivity variations occur primarily with depth. The first term in (6) is the electric field in a uniform-depth ocean whose horizontal components are given by (1), while the second term represents the inductively and galvanically scattered components due to electrical inhomogeneity.

A common procedure for solving (6) is the Born approximation, which requires that the internal electric field be constant across the distorting body, and is obtained by replacing the electric field in the integral term with its background value. This approach involves implicit geometric conditions on the distorting body, and the Born approximation is accurate only when the difference between the internal (to the distorting body) and background electric fields is small. In fact, the Born approximation is the first term in the solution of (6) by successive iteration. This led Habashy et al. (1993) to suggest an extension of the Born method, called the localized nonlinear (LN) approximation, in which the internal electric field behind the integral sign in (6) is replaced with

$$
\mathbf{E}\left(\mathbf{r}^{\prime}\right)=\boldsymbol{\Gamma}\left(\mathbf{r}^{\prime}\right) \mathbf{E}^{o}\left(\mathbf{r}^{\prime}\right),
$$

where the depolarization tensor is given by

$$
\boldsymbol{\Gamma}(\mathbf{r})=\left[\mathbf{I}-\int_{V_{s}} \mathbf{G}\left(\mathbf{r}, \mathbf{r}^{\prime}\right) \delta \gamma\left(\mathbf{r}^{\prime}\right) d \mathbf{r}^{\prime}\right]^{-1} .
$$

Expanding (8) in a Taylor series shows that the LN approximation accounts for multiple internal scattering in the distorting body, leading to an internal electric field that varies spatially. The LN approximation includes a correction for boundary charge-induced variations in the internal electric field, which are not allowed 
under the Born approximation. Habashy et al. (1993) further show that this leads to substantially more accurate solutions under the conditions that apply on Earth. Note that in the galvanic limit that prevails for motional induction, (8) is approximately real and hence the internal and background electric fields will be nearly in phase.

To solve (6) using (7), it is also expedient to assume that the background electric field is uniform across the distorting inhomogeneity and can be approximated at the inhomogeneity by its value at the observation point r. The first condition is equivalent to requiring that the inductive scale in the background medium and the hydrodynamic scale be substantially greater than the largest dimension of the distorting body. The second condition is not a problem for a 1D background model, but might pose difficulties for more complex regional structures. Under these assumptions, (7) is replaced by

$$
\mathbf{E}\left(\mathbf{r}^{\prime}\right)=\boldsymbol{\Gamma}\left(\mathbf{r}^{\prime}\right) \mathbf{E}^{o}(\mathbf{r})
$$

and the background field may be removed from the integral in (6) to give

$$
\mathbf{E}(\mathbf{r}) \approx \mathbf{E}^{o}(\mathbf{r})+\mathbf{F}(\mathbf{r}) \mathbf{E}^{o}(\mathbf{r}),
$$

where

$$
\mathbf{F}(\mathbf{r})=\int_{V_{s}} \mathbf{G}\left(\mathbf{r}, \mathbf{r}^{\prime}\right) \delta \gamma\left(\mathbf{r}^{\prime}\right) \boldsymbol{\Gamma}\left(\mathbf{r}^{\prime}\right) d \mathbf{r}^{\prime}
$$

Equation (10) may be rewritten in the form

$$
\mathbf{E}(\mathbf{r})=\mathbf{C}_{3}(\mathbf{r}) \mathbf{E}^{o}(\mathbf{r}),
$$

where the $3 \times 3$ electric field distortion tensor is given by

$$
\mathbf{C}_{3}(\mathbf{r})=\mathbf{I}+\mathbf{F}(\mathbf{r}) \text {. }
$$

The elements of $\mathbf{C}_{3}(\mathbf{r})$ will be complex unless the inductive parts in (8) or (11) are small compared with the galvanic terms. When this holds, then $\mathbf{C}_{3}(\mathbf{r})$ describes galvanic distortion of the electric field. The distortion tensor elements are also functions of position, and the distortion tensor at one point will generally be different from that at another, depending on the geometry and relative location of the distorting inhomogeneity. This suggests that the distortion parameters contained in $\mathbf{C}_{3}(\mathbf{r})$ are not inherently interesting, but rather serve as an avenue for estimating the undistorted electric field. In practice, only the horizontal components of $\mathbf{E}(\mathbf{r})$ are usually measured, and hence only the upper-left four elements of $\mathbf{C}_{3}(\mathbf{r})$, denoted by $\mathbf{C}(\mathbf{r})$, are of immediate interest.

Note also that (12) or its horizontal components indicate all parts of the background electric field (1) may be galvanically distorted, including the nonlocal current term. This distortion takes the form both of an amplitude shift, which could be parameterized heuristically by altering $\Lambda$, and of directional changes, which cannot be accommodated in the context of (1) and (3). Thus, gal- vanic distortion is not simply a complicated restatement of existing theory, but is a distinct phenomenon that needs to be considered in understanding motional induction data.

\section{Implementation of a tensor decomposition}

The horizontal electric field distortion tensor $\mathbf{C}(\mathbf{r})$ contains four real elements in the galvanic limit, which is expected to prevail for motional induction. Groom and Bailey (1989) showed that the electric galvanic distortion tensor can be uniquely decomposed into the product of a scalar and three tensors:

$$
\mathbf{C}=g \text { TSA, }
$$

where the dependence on location is implicit and $\mathbf{T}, \mathbf{S}$, and $\mathbf{A}$ are called the twist, shear, and anisotropy tensors, respectively, each of which contains a single real parameter in the galvanic limit. The parameter $g$ is a scalar called the site gain. The twist, shear, and anisotropy tensors may be written

$$
\begin{aligned}
\mathbf{T} & =\frac{1}{\sqrt{1+t^{2}}}\left[\begin{array}{cc}
1 & -t \\
t & 1
\end{array}\right], \\
\mathbf{S} & =\frac{1}{\sqrt{1-e^{2}}}\left[\begin{array}{ll}
1 & e \\
e & 1
\end{array}\right], \quad \text { and } \\
\mathbf{A} & =\frac{1}{\sqrt{1-s^{2}}}\left[\begin{array}{cc}
1+s & 0 \\
0 & 1-s
\end{array}\right] .
\end{aligned}
$$

The normalizing factors in (15)-(17) have been chosen to make the tensor forms symmetric with respect to their inverses. Physically, the twist tensor is a rotation that takes the regional electric field through a clockwise angle $\phi_{t}=\tan ^{-1} t$ to yield the observed (distorted) electric field, where $\phi_{t}$ is called the twist angle. The shear tensor develops anisotropy along axes that bisect the principal axes of the regional electric field, and may be characterized by the shear angle $\phi_{e}=\tan ^{-1} e$. The maximum angular change occurs for vectors aligned along the principal axes; a vector on the $x$ axis is deflected counterclockwise through $\phi_{e}$ while a vector on the $y$ axis is deflected clockwise by the same amount. The anisotropy tensor scales the two electric field components by different factors. Groom and Bailey (1989; see their Figs. 2 and 3) give a physical picture of the effect of the shear and anisotropy tensors. Finally, the site gain performs an overall amplitude change on the electric field. In practice, the scalars in front of the matrices in (15)-(17) may be subsumed into the site gain $g$ to simplify subsequent algebra.

In the event that a series of point measurement sites are occupied, a suitable way to get the distortion parameters is to consider the intersite electric field transfer functions at high frequencies where the electric field is dominated by long wavelength (compared to the spacing of the observation sites) magnetospheric and ionospher- 
ic sources. Let $\mathbf{Z}_{i j}$ denote the transfer function or impedance tensor relating the electric fields between the $i$ th and $j$ th site. Here, $\mathbf{Z}_{i j}$ contains at most four complex (or eight real) parameters at each frequency. Let $\mathbf{Z}_{i j}^{o}$ denote the model transfer function relating the background fields at the $i$ th and $j$ th site. In the absence of galvanic distortion and for a uniform source field, this quantity will be the identity matrix because the electric field is a measure of electric current flowing near the seafloor and the conductivity of seawater is isotropic. In general, it could be a complex diagonal matrix containing four real parameters at each frequency, although it is expected that its elements will be frequency independent if the galvanic model is correct. The phase of the complex elements is a measure of the spatial variation of the source field and should be close to zero if the site spacing is small compared to the dominant source field wavelength. In the sequel, $\mathbf{Z}_{i j}^{o}$ will be taken to be a frequency-independent complex diagonal matrix containing at most four real parameters, and the validity of the model will be assessed by examining the model fit to the data.

Substituting from the definition of the transfer functions, it is easy to show that

$$
\mathbf{Z}_{i j}=\mathbf{C}_{i} \mathbf{Z}_{i j}^{o} \mathbf{C}_{j}^{-1} .
$$

Substituting (14) into (18) yields

$$
\mathbf{Z}_{i j}=\frac{g_{i}}{g_{j}} \mathbf{T}_{i} \mathbf{S}_{i} \mathbf{A}_{i} \mathbf{Z}_{i j} \mathbf{A}_{j}^{-1} \mathbf{S}_{j}^{-1} \mathbf{T}_{j}^{-1} .
$$

This presumes that $\mathbf{Z}_{i j}^{o}$ is in its principal axes, which will be approximately true if the electric fields at sites $i$ and $j$ have the same geographic orientation. Note that instrument orientation problems (such as would occur from compass error) are indistinguishable from distortion-induced twist since the twist tensor represents a rotation. In practice, any required diagonalization of $\mathbf{Z}_{i j}^{o}$ can be subsumed into the twist tensors and hence will be ignored.

Further simplification of (19) is facilitated using a Pauli spin matrix basis. Let the Pauli spin basis consist of the four matrices

$$
\begin{aligned}
& \mathbf{I}=\left[\begin{array}{ll}
1 & 0 \\
0 & 1
\end{array}\right], \quad \boldsymbol{\Sigma}_{1}=\left[\begin{array}{ll}
0 & 1 \\
1 & 0
\end{array}\right], \\
& \boldsymbol{\Sigma}_{2}=\left[\begin{array}{cc}
0 & -1 \\
1 & 0
\end{array}\right], \quad \text { and } \quad \boldsymbol{\Sigma}_{3}=\left[\begin{array}{cc}
1 & 0 \\
0 & -1
\end{array}\right] \text {. }
\end{aligned}
$$

It is easy to derive the multiplicative algebra of this basis. We may expand a general $2 \times 2$ matrix or secondrank tensor uniquely as

$$
\mathbf{B}=B_{0} \mathbf{I}+B_{1} \boldsymbol{\Sigma}_{1}+B_{2} \boldsymbol{\Sigma}_{2}+B_{3} \boldsymbol{\Sigma}_{3}
$$

where

$$
\begin{array}{ll}
B_{0}=\frac{b_{11}+b_{22}}{2}, & B_{1}=\frac{b_{12}+b_{21}}{2}, \\
B_{2}=\frac{b_{21}-b_{12}}{2}, & \text { and } \quad B_{3}=\frac{b_{11}-b_{22}}{2} .
\end{array}
$$

Using the multiplicative algebra, we may expand the terms in (19) in the Pauli basis. First, we represent the central part of (19) as

$$
\mathbf{A}_{i} \mathbf{Z}_{i j}^{o} \mathbf{A}_{j}^{-1}=\alpha \mathbf{l}+\beta \mathbf{\Sigma}_{3},
$$

where $\alpha=\rho\left(1-s_{i} s_{j}\right)+\delta\left(s_{i}-s_{j}\right), \beta=\rho\left(s_{i}-s_{j}\right)+$ $\delta\left(1-s_{i} s_{j}\right), \rho=(a+b) / 2, \delta=(a-b) / 2$, and $a$ and $b$ are the respective upper and lower elements of the diagonal tensor $\mathbf{Z}_{i j}^{o}$. Writing out the terms in (23) gives

$$
\mathbf{A}_{i} \mathbf{Z}_{i j} \mathbf{A}_{j}^{-1}=\left[\begin{array}{cc}
\left(1+s_{i}\right)\left(1-s_{j}\right) a & 0 \\
0 & \left(1-s_{i}\right)\left(1+s_{j}\right) b
\end{array}\right],
$$

which is indistinguishable from the original $\mathbf{Z}_{i j}^{o}$. Thus, we cannot uniquely differentiate distortion-induced and structural anisotropy. We can choose to ascribe all of the observed anisotropy in $\mathbf{Z}_{i j}^{o}$ to distortion, in which case $a$ and $b$ are complex numbers with a modulus of 1 to account for source field variation. In the subsequent development, we will carry the full form (23) through, although it must be recognized that this contains only two magnitude parameters (either the moduli of $a$ and $b$ or the two anisotropy parameters) plus two phase factors.

Expanding the remainder of (19) and ignoring the scale factors $g_{i}$ and $g_{j}$ (which are indeterminate without additional data or assumptions) gives

$$
\begin{aligned}
\mathbf{T}_{i} \mathbf{S}_{i} \mathbf{A}_{i} \mathbf{Z}_{i j} \mathbf{A}_{j}^{-1} \mathbf{S}_{j}^{-1} \mathbf{T}_{j}^{-1} \\
=\left[\alpha\left(1-e_{i} e_{j}\right) \cos \left(\varphi_{i}-\varphi_{j}\right)\right. \\
\left.-\beta\left(e_{i}+e_{j}\right) \sin \left(\varphi_{i}-\varphi_{j}\right)\right] \mathbf{l} \\
+\left[\alpha\left(e_{i}-e_{j}\right) \cos \left(\varphi_{i}+\varphi_{j}\right)\right. \\
\left.+\beta\left(1+e_{i} e_{j}\right) \sin \left(\varphi_{i}+\varphi_{j}\right)\right] \mathbf{\Sigma}_{1} \\
+\left[\alpha\left(1-e_{i} e_{j}\right) \sin \left(\varphi_{i}-\varphi_{j}\right)\right. \\
\left.\quad+\beta\left(e_{i}+e_{j}\right) \cos \left(\varphi_{i}-\varphi_{j}\right)\right] \mathbf{\Sigma}_{2} \\
-\left[\alpha\left(e_{i}-e_{j}\right) \sin \left(\varphi_{i}+\varphi_{j}\right)\right. \\
\left.\quad-\beta\left(1+e_{i} e_{j}\right) \cos \left(\varphi_{i}+\varphi_{j}\right)\right] \mathbf{\Sigma}_{3},
\end{aligned}
$$

where $\phi_{i}=\tan ^{-1} t_{i}$. The measured transfer tensor $\mathbf{Z}_{i j}$ may be expanded in the Pauli spin basis using (21)(22). This yields four equations for each transfer function containing eight unknowns at each frequency (the pairs of shear and twist parameters, either the moduli of $a$ and $b$ or the pair of anisotropy factors at each site, and two phase factors at each site). The equations can be solved by nonlinear least squares over all available frequencies either for a pair of sites (i.e., one transfer function), or simultaneously over multiple sites, by re- 
quiring that the distortion parameters be frequency independent, as expected for purely galvanic distortion. In either case, the rotation represented by the twist tensor is modulo $\pi / 2$ and insensitive to an arbitrary rotation applied to all sites. This ambiguity may be resolved if the data are initially oriented within $90^{\circ}$ and if there is at least one reference site whose absolute orientation is known. Once the distortion parameters are available, and remembering that they are frequency independent, the undistorted electric field follows by inverting (12). Since the frequency-domain distortion parameters are frequency independent, they may be applied in the time domain very easily. The validity of the overall procedure and the assumptions that underlie it can then be assessed statistically.

This procedure only yields the electric field to within a pair of multiplicative constants (the site gains $g_{i}$ and $g_{j}$ ) applied independently to the two horizontal components. The constants cannot be determined from electric field data alone, and must be derived from other physical assumptions or independent measurements of the underlying hydrodynamic phenomena. For example, fixed-point sensor electric field measurements might be calibrated against densely instrumented current meter moorings, profiler data, or some other adequate measure of the vertically averaged water velocity. Geophysical calibration is also feasible; if the spatial variation of the externally induced electric field amplitude at high frequencies is understood, it can be used to scale the electric field.

\section{Application to the SAFDE dataset}

The SAFDE instruments and data have been described in section 2 and Table 1 . The electrometers are oriented by recording the image of a suspended and gimballed compass on photographic film, hence yielding both azimuth and vector tilt information. Instrument tilts were modest (a few degrees) at all sites except H04 $\left(19^{\circ}\right), \mathrm{H} 10\left(19^{\circ}\right)$, and H16 (where it exceeded $25^{\circ}$, the maximum tilt that can be measured). Two of the instruments (H13 and H14) showed clear evidence of instrument rotation during the first $1 / 3$ of the record because of multiple compass images, presumably due to very intense bottom current events. This was easily detectable in the data as an abrupt shift in the baseline, and a differential rotation was applied to remove it. Based on the intensity of the compass image, H14 was tilted only slightly while $\mathrm{H} 13$ displays a $22^{\circ}$ value over most of the record. Instrument debriefing was further complicated by compass failure at all sites due to the very high $\left(\sim 80^{\circ}\right)$ inclination of the geomagnetic field at the SAFDE latitude. As a result, rough alignment of the data was accomplished by visual comparison and vector spectral analysis between sites and with a nearby standard geomagnetic observatory. This result is believed to be accurate to $10^{\circ}-20^{\circ}$.

Intersite transfer tensors between the two horizontal
TABLE 2. Final distortion parameters.

\begin{tabular}{lccc}
\hline \hline Site & Anisotropy & Shear $\left(^{\circ}\right)$ & Twist $\left(^{\circ}\right)$ \\
\hline H02 & 0.0967 & -3.6 & -11.7 \\
H03 & 0.1876 & -7.5 & -11.5 \\
H04 & 0.1017 & -2.4 & -11.5 \\
H07 & 0.0900 & 1.0 & -11.7 \\
H08 & -0.0444 & -3.2 & -11.7 \\
H09 & -0.2077 & -2.3 & -11.9 \\
H10 & 0.1653 & 1.5 & -11.3 \\
H11 & -0.1682 & 0.3 & -12.1 \\
H12 & 0.0075 & -5.9 & -11.8 \\
H13 & 0.2242 & 2.3 & -11.1 \\
H14 & 0.0154 & -0.9 & -11.0 \\
H15 & -0.0654 & 3.5 & -11.4 \\
H16 & -0.3034 & 8.4 & -11.7 \\
H17 & -0.1099 & 2.0 & -11.4 \\
\hline
\end{tabular}

electric field components at adjacent pairs of sites were computed from north to south along the line from $\mathrm{H} 02$ to $\mathrm{H} 17$, yielding a total of 13 transfer tensors. Because the solar daily variation or its harmonics possess a continuum component at periods longer than $4 \mathrm{~h}$ that has source wavelength characteristics that are quite different from the remaining external sources, attention was focused on the transfer tensor values at periods of $3 \mathrm{~h}$ to $15 \mathrm{~min}$. In this band, the electric field variations are dominated by auroral substorm activity, which has a relatively simple spatial structure. However, the time series are quite nonstationary, and the remote reference bounded influence algorithm of Chave and Thomson (2003a,b) was used to minimize bias from extreme outliers and leverage points (which do not necessarily correspond to the most energetic events), resulting in elimination of almost half of the data. For the same reason, use of the jackknife (Thomson and Chave 1991) is advised to compute standard errors on the tensor elements. The resulting transfer tensor elements possess 5000-40 000 equivalent degrees of freedom at each of eight frequencies, and are smooth functions that are nearly independent of frequency. These constitute the data for input to the tensor decomposition (25).

The tensor decomposition (25) under the assumption that the underlying transfer tensor is isotropic [so that the magnitudes of $a$ and $b$ in (24) are assumed to be due solely to distortion anisotropy, and hence set to 1] was applied simultaneously to all of the transfer tensors, used to identify the individual rotation (twist) angles for each site, and then the time series were rotated to the new coordinate system. New transfer tensors were computed, and this process continued iteratively until no further significant $\left(>1^{\circ}\right)$ rotations were required. For the final iterations, the sites were aligned deleting $\mathrm{H} 12$ and $\mathrm{H} 15$, where the records are short and hence the transfer tensor uncertainties are commensurately large. After alignment of the remaining data, H12 and H15 were decomposed with the distortion model for the remaining sites fixed. Table 2 gives the final distortion parameters for each site. The mean twist with $\mathrm{H} 12$ and $\mathrm{H} 15$ deleted is $-11.6^{\circ}$, and all entries are within $0.6^{\circ}$ 
TABLE 3. Total $\chi^{2}$ misfits.

\begin{tabular}{lcrr}
\hline \hline Site 1 & Site 2 & $\sum_{i=1}^{8} \chi_{i}^{2}$ & $\chi_{(4)}^{2}$ \\
\hline H02 & H03 & 111.9 & 11.1 \\
H03 & H04 & 79.2 & 9.3 \\
H04 & H07 & 125.6 & 12.5 \\
H07 & H08 & 70.8 & 7.3 \\
H08 & H09 & 47.5 & 5.5 \\
H09 & H10 & 47.0 & 6.3 \\
H10 & H11 & 49.7 & 4.7 \\
H11 & H13 & 102.6 & 11.8 \\
H12 & H15 & 51.2 & 3.7 \\
H13 & H14 & 40.8 & 4.0 \\
H14 & H16 & 96.0 & 11.6 \\
H15 & H16 & 47.4 & 3.0 \\
H16 & H17 & 62.0 & 5.0 \\
\hline
\end{tabular}

of this value. The actual mean value of the twist is not critical, as an arbitrary rotation may be applied to all sites in the absence of compass information without altering the decomposition terms.

Table 3 gives the total $\chi^{2}$ misfit over the eight frequencies in the transfer tensors between the listed sites. Except at the end points of the line, for each pair of sites, there are 64 data ( 8 data at eight frequencies) and 19 parameters (two phases at each frequency plus half of three frequency-independent distortion parameters at each of two sites), yielding 45 equivalent degrees of freedom. At the end points, this decreases to $43.5 \mathrm{de}-$ grees of freedom, as three of the frequency-independent parameters are estimated from only a single transfer tensor estimate. The expected value for $\chi^{2}$ is 45 (43.5 at the end points) and the $95 \%$ point is 61.6 (60.5 at the end points). Using a $95 \%$ criterion on the total $\chi^{2}$, the fit is not acceptable for seven (or just over half) of the transfer tensors, and the distortion model would appear to be only marginally appropriate. However, total $\chi^{2}$ is notoriously lacking in robustness, being very sensitive to even a single outlying value, and perusal of the misfits by frequency for those sites with high $\chi^{2}$ indicates that they are often biased by one or two extreme values. A robust alternative would be the median value of $\chi^{2}$. For eight frequencies, this quantity is not well defined, but a reasonable approach entails ordering the data and taking the fourth value; this is called the fourth-order statistic and is given in the last column of Table 3. The sampling pdf of the $r$ th-order statistic from a sample of size $N$ is given by (Stuart and Ord 1994, chapter 14)

$$
g\left[x_{(r)}\right]=\frac{\left\{F\left[x_{(r)}\right]\right\}^{r-1}\left\{1-F\left[x_{(r)}\right]\right\}^{N-r} f\left[x_{(r)}\right]}{B(r, N-r+1)},
$$

where $x_{(r)}$ is the $r$ th-order statistic, $B$ is the beta function, and $f(x)$ and $F(x)$ are the underlying probability density function (pdf) and cumulative distribution function, respectively. For this problem, $N=8, r=4$, and $f(x)$ and $F(x)$ may be taken as $\chi_{6}^{2}$. Numerical evaluation gives a $95 \%$ point for this parameter of about 7.4. Using a 95\% criterion, Table 3 shows an acceptable value for all except the H02-H03, H03-H04, H04-H07, H11$\mathrm{H} 13$, and H14-H16 transfer tensors. Note that the last three of these site pairs are more widely $(70-100 \mathrm{~km})$ spaced than the remaining datasets, and this separation approaches the distance to the auroral electric currents producing the observed electric field variations. Further, the $\mathrm{H} 02-\mathrm{H} 03$ and $\mathrm{H} 03-\mathrm{H} 04$ sites are also physically distant from the bulk of the data. As a result, the assumption of source field homogeneity is weakest for all of these pairs of sites and may account for the lingering discrepancy.

A remaining and unquantified problem is the size of the galvanically induced twist, which acts as a source of error for estimating the instrument orientation in the absence of working instrument compasses. To place a bound on twist, a set of electric field data from the Mantle Electromagnetic and Tomography (MELT) experiment (Evans et al. 1999) collected in a geologically similar environment crossing the East Pacific Rise and for which the instrument compasses were fully functional was decomposed. For four closely spaced sites on the north MELT line, a mean twist difference of about $2^{\circ}$ was obtained. This strongly suggests that the procedure applied to the SAFDE data yields reliable instrument orientations, and that galvanic twist is not an appreciable source of error or bias.

As a consistency check, the distortion parameters in Table 2 were applied to each of the time series to remove the galvanic distortion and align them in a relative sense. The electric field transfer tensors were then recomputed, yielding in each instance an isotropic diagonal tensor multiplied by a scalar to within the jackknife error estimates.

As previously noted, independent information is required to estimate the site gains and an absolute azimuthal reference for at least one site is required to orient all of the sites. Ideally, the comparison would be with an independent estimate of the CWVI velocity that is measured by the HEF. An estimate of that quantity was obtained using other data that were collected at approximately the same location as H09. Inverted echo sounder (IES) measurements of round-trip travel time were combined with historical hydrography from the region using the gravest empirical mode (GEM) technique to produce time series of full water column profiles of temperature, salinity, and specific volume anomaly following the methods of Meinen and Watts (2000). By integrating the profiles of the specific volume anomaly, estimates of the profile of the geopotential height anomaly were obtained. Differencing coincident profiles from neighboring sites yields an estimate of the geostrophic velocity relative to the bottom at H09. Further details may be found in Meinen et al. (2002). This relative velocity has to be referenced to provide an absolute velocity for comparison with HEF-inferred velocity. A current meter at a nominal depth of $2000 \mathrm{~m}$ and located about $1 \mathrm{~km}$ from H09 provides the necessary absolute velocity. Temperature measurements from this current 
meter were combined with the temperature profiles determined from the IES-GEM technique to provide an estimate of the blowover of the mooring (Meinen and Luther 2002), and the resulting time series of pressure and velocity were used to reference the relative velocity profile from the IES-GEM measurements. The temperature and salinity estimates obtained from the IES-GEM method were also used to calculate full water column time series of conductivity at the same site. These conductivity profiles were combined with the absolute velocity profiles and integrated over the water column to obtain an estimate of the CWVI absolute velocity.

The H09 velocity data were low passed with a 72-hcutoff fourth-order Butterworth filter applied forward and backward over the data to avoid phase shifts. These data were then oriented to a comparably low-passed version of the IES-GEM product through the least squares solution over the whole time series of

$$
\begin{aligned}
& \bar{u}=A \cos \theta\langle u\rangle^{*}+B \sin \theta\langle v\rangle^{*} \text { and } \\
& \bar{v}=-A \sin \theta\langle u\rangle^{*}+B \cos \theta\langle v\rangle^{*},
\end{aligned}
$$

where $\bar{u}$ and $\bar{v}$ are the zonal and meridional CWVI absolute velocities from the IES-GEM product, $\langle u\rangle^{*}$ and $\langle v\rangle^{*}$ are estimates of these velocities from the HEF data after distortion removal, $A$ and $B$ are scalars, and $\theta$ is a rotation angle. This problem is nonlinear, but the normal equations decouple so that it is easily solved iteratively. Convergence is achieved after six iterations, yielding $A=1.1016, B=1.0943$, and $\theta=-10.3^{\circ}$. Note that $A$ and $B$ are identical to within $0.7 \%$, in good agreement with galvanic distortion theory, and the scalar site gain may be taken as their average. Figure 4 compares the IES-GEM velocity product with the rotated and scaled HEF data. The rms misfits for the zonal and meridional components are 2.3 and $1.7 \mathrm{~cm} \mathrm{~s}^{-1}$, respectively, which is well within the estimation error.

The agreement of the IES-GEM- and HEF-derived CWVI velocity estimates indicates that nonlocal electric currents at site H09 are quite small. Based on the rms misfits from the time series compared in Fig. 4, they cannot exceed $2 \mathrm{~cm} \mathrm{~s}^{-1}$, and in fact a value this large would require that the IES-GEM product be regarded as an error-free measurement of the CWVI velocity. The relative insignificance of nonlocal electric currents in the H09 data, combined with the agreement of the amplitude fluctuations in the auroral and motional induction frequency bands (see section 2 and Fig. 2), strongly suggests that nonlocal electric currents are small in the SAFDE data as a whole.

The remaining time series at $\mathrm{H} 02-\mathrm{H} 08$ and $\mathrm{H} 10-\mathrm{H} 17$ were rotated through a $10.3^{\circ}$ clockwise angle to yield final datasets exclusive of the site gains. Independent estimates for water velocity are not available at any of these locations for amplitude calibration purposes. However, external (ionospheric and magnetospheric) electric current systems are sufficiently well characterized that amplitude scale factors may be derived from the vari-
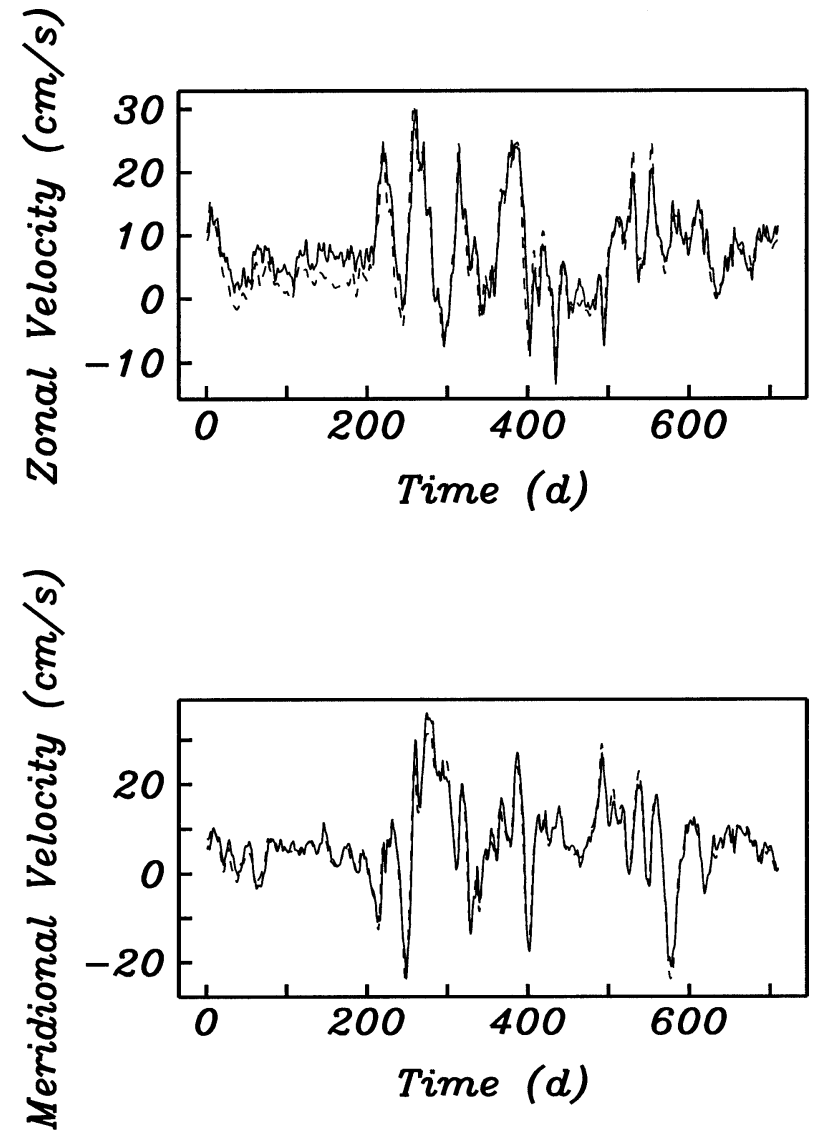

FIG. 4. Comparison of the conductivity weighted, vertically averaged velocity product at $\mathrm{H} 09$ estimated from mooring and IES data as described in the text (solid lines) with the measured velocities from HEF data (dashed lines) from the same site after rotation and scaling by solving (27). Both datasets have been low-pass filtered with a 72$\mathrm{h}$ cutoff. The rms misfit is (top) $2.3 \mathrm{~cm} \mathrm{~s}^{-1}$ for the zonal component and (bottom) $1.7 \mathrm{~cm} \mathrm{~s}^{-1}$ for the meridional component.

ation of high-frequency ( $<1 \mathrm{cpd}$ ) energy with location. Campbell (1997, chapter 3) provides a recent overview of the characteristics and physics of external current systems. While there are a number of candidates, at high latitudes the most energetic (albeit highly episodic) external source is the polar or auroral substorm.

It has long (e.g., Chapman 1935) been known that the surface electromagnetic signatures of two- and threedimensional external current systems are indistinguishable, but seminal work by Boström (1964) has shown that the electric currents associated with auroral substorms are in fact complex and three-dimensional. The primary current loop consists of an ionospheric electrojet at altitudes of $O(100 \mathrm{~km})$ whose electromagnetic effects dominate at Earth's surface linked to the outer magnetosphere at many earth radii by field-aligned (Birkeland) currents. There are two quasi-independent modes for electric current flow associated with an eastwest and north-south electrojet, respectively. The former occurs primarily during substorms and involves much higher ionospheric electric currents and a stronger 
surface electromagnetic signature than the north-south mode. Further characteristics of auroral substorms are reviewed by Kisabeth and Rostoker (1977), Nishida (1978), Hughes and Rostoker (1979), Rostoker and Hughes (1979), and Mareschal (1981, 1986). A principal feature is a dominantly east-west substorm ionospheric current system with a stable equatorward boundary and a poleward boundary that intensifies and expands nearly explosively toward the pole, followed by amplitude decay accompanied by equatorward current system motion. The characteristic time scale ranges from hours to (rarely) days. The electrojet is largely confined to a variable auroral oval ranging nominally from $66^{\circ}$ to $78^{\circ}$ geomagnetic latitude, or about $2400-$ $1200 \mathrm{~km}$ from the dip pole where Earth's magnetic field is vertical. In the absence of galvanic distortion, the surface electric field from auroral substorms will be dominantly polarized east-west relative to the dip pole. Further, the statistically averaged surface electric field amplitude from auroral substorms within the auroral oval will exhibit a general increase toward the dip pole, falling off more rapidly on either side.

For calibration purposes, it is important to utilize a portion of the frequency spectrum that is dominated by a single source process whose variability is understood. Substorm fields are not strongly mixed with those from other sources at periods shorter than about $4 \mathrm{~h}$ (above which the solar daily variation and its harmonics become important) and longer than about $1 \mathrm{~h}$ (below which various hydromagnetic wave sources become important), and hence this part of the spectrum will be the principal focus. It is also important to consider an adequately long statistical average of the field, as individual substorm events can be highly variable. Figure 5 shows power spectra for the H09 final data computed from typical 4-day ionospherically active (i.e., substorm dominated) and quiet (i.e., nonsubstorm time) sections, respectively. The spectral slope is approximately $1 / f^{2}$ from $0.1-2 \mathrm{cph}$ (cph $\equiv$ cycles per hour) and increases at higher frequencies for both active and quiet times, suggesting a change of process above $\sim 1 \mathrm{cph}$. Note that the meridional power (which corresponds to the eastwest electric field) is a factor of 5-10 larger than zonal power when auroras are present, suggesting that the underlying electric field is, as expected, strongly polarized east-west. Note also the high degree of nonstationarity, with the power spectral density increasing by one to two decades during substorm (active) times. Substorms are visually apparent in the time series for $1 / 3-$ $1 / 2$ of the record, comprising over 100 discrete events. This strongly suggests that statistical averages computed from the time series will be representative of average auroral substorm activity.

The HEF time series were bandpass filtered using a four-pole Butterworth filter having cutoffs at 3.8 and $2.5 \mathrm{~h}$, respectively. The standard deviation of each filtered time series was then computed, and appears in Fig. 6 plotted against the distance from each site to
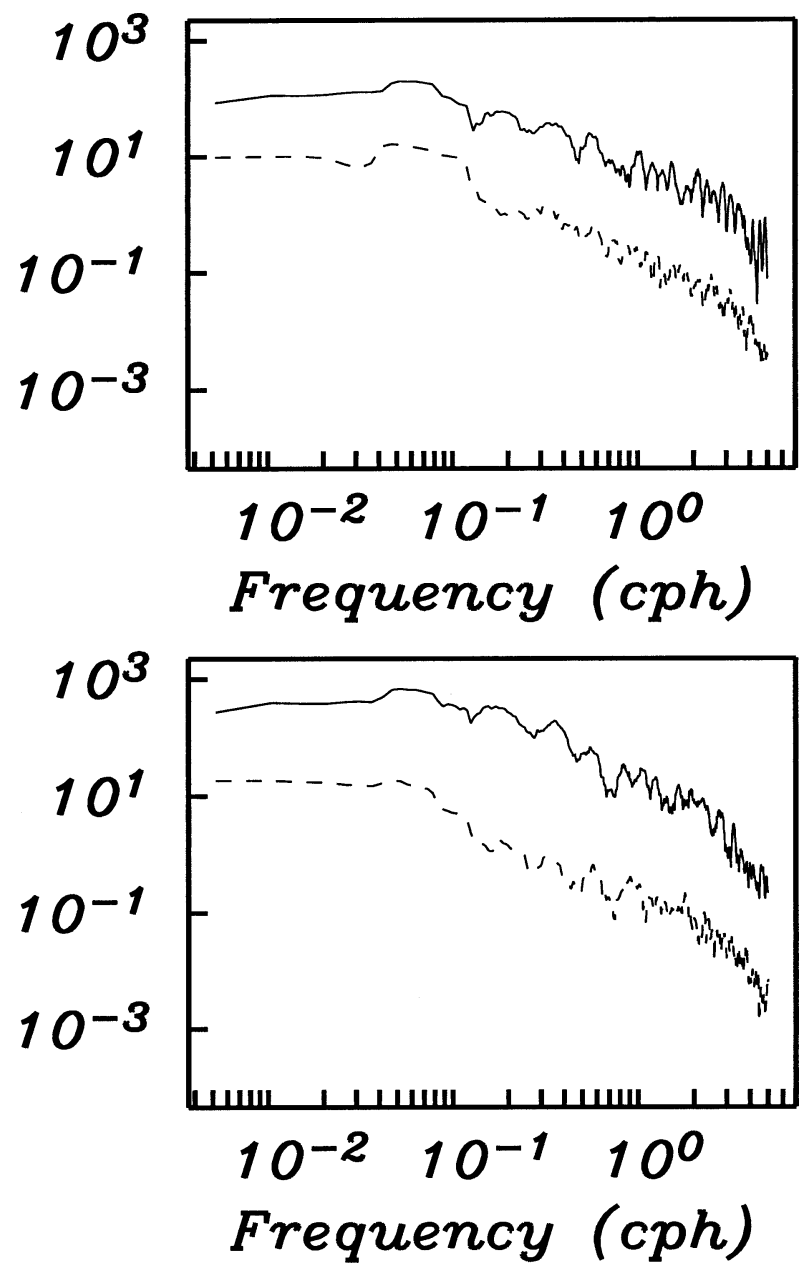

FIG. 5. Power spectra $\left[\left(\mathrm{cm} \mathrm{s}^{-1}\right)^{2}(\mathrm{cph})^{-1}\right]$ for 4-day-long active (solid line) and quiet (dashed line) pieces of the (top) zonal and (bottom) meridional time series for $\mathrm{H} 09$ after final rotation and scaling as described in the text. All of the spectra were computed identically using the multitaper method with a time bandwidth of four and eight prolate windows, yielding about 14 degrees of freedom per frequency and a bandwidth of about $0.02 \mathrm{cph}$.

Earth's southern dip pole $\left[64.7^{\circ} \mathrm{S}, 139.2^{\circ} \mathrm{E}\right.$ for the 1995 epoch; Macmillan et al. (1997)], which serves as a proxy for substorm energy. Serendipitously, the SAFDE array is oriented within $5^{\circ}$ of the geographic north-south great circle passing through the dip pole, and hence the zonal and meridional velocities estimated using (3) nearly represent the geomagnetic north-south and east-west electric fields, respectively. The time mean electric field is strongly polarized along $102^{\circ}-282^{\circ}$ true, consistent with this observation and the behavior expected of auroral substorms. Although the data are scattered, both the zonal and meridional variances increase in an approximately linear fashion as the dip pole is approached. Since the length of the SAFDE array (about $400 \mathrm{~km}$ ) is a fraction of the size of the auroral oval (about 1200 $\mathrm{km}$ ), approximately linear amplitude change would be expected even if there is some curvature in the substorm 


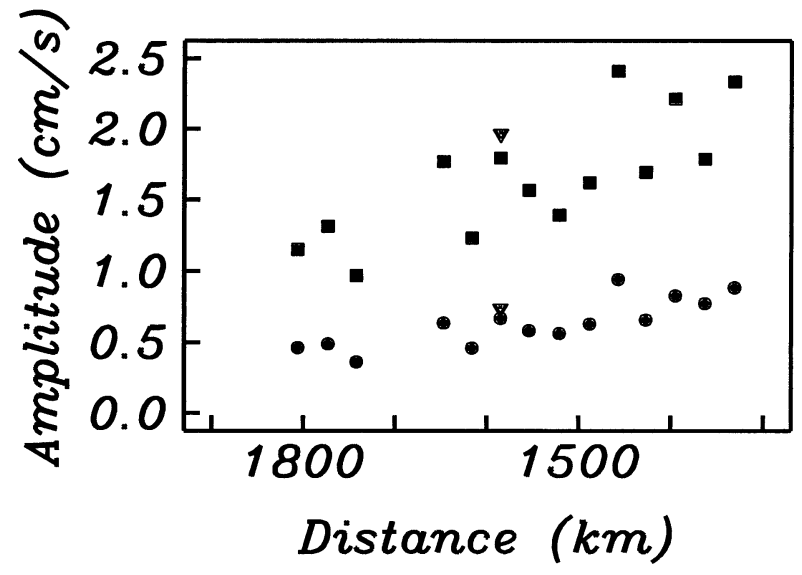

FIG. 6. Std dev of the zonal (solid circles) and meridional (solid squares) time series after final rotation and bandpass filtering over $3.8-2.5 \mathrm{~h}$ plotted against the distance to Earth's dip pole in $\mathrm{km}$. The inverted triangles show the amplitude scaled with the IES-GEM product at site $\mathrm{H} 09$, while the corresponding circle and square for that site are unscaled $[A=B=1$ in (27)].

amplitude-geomagnetic latitude relationship. Further, the relative scatter in the zonal and meridional components is comparable, as expected if it is due to galvanic distortion.

Regression lines were fit to the zonal and meridional standard deviation data of Fig. 6 after excluding H12 and $\mathrm{H} 15$ due to the brevity of those records and utilizing the unscaled data at $\mathrm{H} 09$ for consistency. Examination of the residuals and hat matrix diagonals reveals no serious outliers or leverage points, respectively. The ratio of residual variance (estimate of 7.94) was tested against double-sided $F_{10,10}$ (critical value of 3.72), and the null hypothesis that the two components share a common variance was rejected at the $95 \%$ significance level. Due to the Behrens-Fisher problem (Stuart et al. 1999, chapter 19), this precludes further testing of the slopes and intercepts to assess their distinctness and hence determine if a single line can be fit simultaneously to the zonal and meridional data, which would yield the site gain for each location if the line were forced to pass through the scaled value at H09. It would be possible to utilize separate calibration lines for the zonal and meridional components by forcing each line to pass through the respective $\mathrm{H} 09$ calibration point, but the resulting site gain would not necessarily be scalar as expected from theory. A scalar site gain could be forced by using the mean of the zonal and meridional values. However, the meridional energy is much larger than the zonal energy, so the former mean is more reliable and the zonal component is correspondingly sensitive to slight orientation errors; a $1^{\circ}$ error in the mean orientation would result in an $8 \%$ error in the zonal amplitude but only a $0.4 \%$ error in the meridional amplitude. Further, the high-frequency zonal and meridional variations are highly correlated, so separate calibrations make little statistical sense. This approach gives undue weight to
TABLE 4. Final amplitude scale factors.

\begin{tabular}{lc}
\hline \hline Site & Scale factor \\
\hline H02 & 1.2756 \\
H03 & 1.1838 \\
H04 & 1.6738 \\
H07 & 1.0396 \\
H08 & 1.5409 \\
H09 & 1.0000 \\
H10 & 1.2993 \\
H11 & 1.4987 \\
H13 & 1.3378 \\
H14 & 0.9262 \\
H15 & 1.3582 \\
H16 & 1.0750 \\
H17 & 1.3442 \\
\end{tabular}

the less reliable zonal estimate, and was deemed unsuitable. Instead, the magnitude was formed from the zonal and meridional standard deviations and regressed on distance to the dip pole. The residuals and hat matrix diagonals were found to be outlier and leverage point free. The scalar site gains follow by requiring the magnitude of each filtered velocity field to lie on the line with the regression slope that passes through the scaled H09 magnitude and are given in Table 4. The standard deviations of Fig. 6 scaled using these estimates appear in Fig. 7 and show smooth variation with distance to the dip pole with an obvious deviation from linearity only for the zonal component at H16.

\section{Discussion}

Galvanic distortion of the electric (and sometimes magnetic) fields is ubiquitous in terrestrial magnetotelluric studies, and correction for its effects has become a routine data processing step. Static shifts (represented by the site gain) of a factor of 2 would not be considered unduly large by this community. While the seafloor

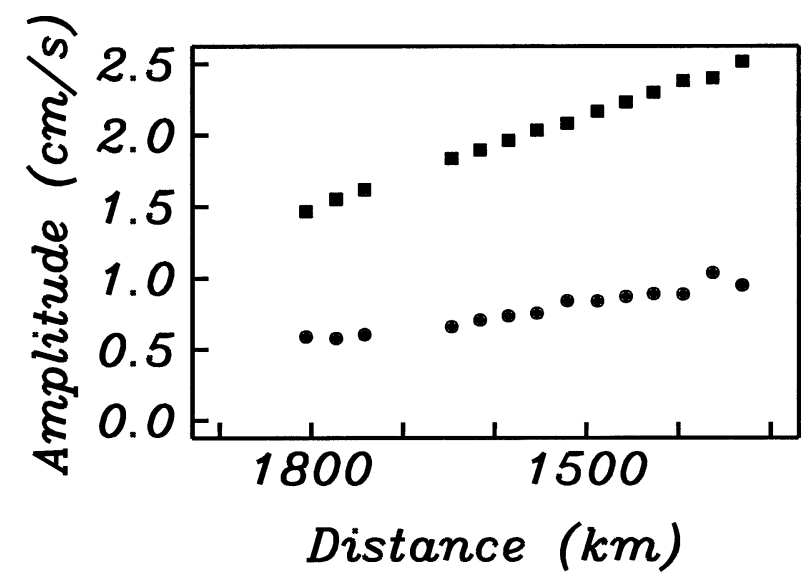

FIG. 7. Std dev of the zonal (solid circles) and meridional (solid squares) time series after bandpass filtering over 3.8-2.5 $\mathrm{h}$ and scaling plotted against the distance to Earth's dip pole in $\mathrm{km}$. 
magnetotelluric database is considerably smaller, recent studies document the pervasive influence of electromagnetic distortion, especially in regions of rough, nearly sediment-free topography such as the Tahiti swell (Nolasco et al. 1998) and the East Pacific Rise (Evans et al. 1999). There is evidence for significant static shift even in sedimented regions and at large scales; Lizarralde et al. (1995) document a $40 \%$ shift in the electric field amplitude from a magnetotelluric study using an abandoned submarine telephone cable extending from California to Hawaii. Since the electric charges that cause galvanic distortion accumulate where strong electrical conductivity gradients exist, it is reasonable to expect that a thick sediment blanket will mitigate its effects to some degree. This may be the reason why static shifts in the motional electric field as large as those seen in Fig. 6 have not previously been documented, but should not be taken as a guarantee.

While the importance of galvanic distortion will have to be assessed on a case-by-case basis, it is prudent to incorporate its correction into experimental planning when electric field measurements are included. This requires consideration of both amplitude (as parameterized by the anisotropy and site gain parameters) and directional (as parameterized by shear and twist) distortion. Amplitude distortion can easily be corrected if one or more independent measurements of the vector velocity and temperature (hence electrical conductivity) fields are available from profilers, IESs with absolute velocity referencing, or other direct means. In situ calibration of the HEF imposes a requirement that is analogous to the selection of a reference level in hydrography or the calibration of IESs using either CTD profiles (Tracey et al. 1997) or historic hydrography combined with absolute pressure measurement (Meinen and Watts 1998). As an alternative, geophysical calibration can be employed as in the present work, although its accuracy is less easily quantified. In fact, the calibration requirement does not represent a change from prior practice where the scale factor $\Lambda$ in (1) and (3) depends on the electrical structure beneath the seafloor and must be estimated from other data. However, in the presence of galvanic distortion, the effective $\Lambda$ will be site dependent, and hence estimates based on regional sediment thickness or average seafloor conductivity will not be sufficient. It is recommended that the previous common practice of attributing nonunity $\Lambda$ to seafloor sediments be discontinued, as the scale factor on the motional electric field depends on more complicated physical phenomena than simple electric current leakage.

Directional distortion cannot be detected or corrected by this calibration procedure as easily as amplitude distortion, and recourse to the tensor decomposition approach is recommended whenever feasible. For the SAFDE data, directional distortion is nontrivial; in the worst case, shear imposes a $15^{\circ}$ shift in the field direction, with a corresponding amplitude shift.

In this paper, the galvanic distortion problem and its correction have been targeted explicitly at fixed (i.e., seafloor) HEF measurements. However, galvanic distortion is an issue for both profiler and towed measurements of the HEF, and should be considered in interpreting such data. Further assessment of its importance and possible correction are future issues.

Acknowledgments. SAFDE has been supported by NSF Grants OCE-9204063 and OCE-9912110 (WHOI), and OCE-9204113 and OCE-9911974 (University of Hawaii).

\section{REFERENCES}

Bailey, J. W., E. Hobart, H. H. Moeller, J. H. Filloux, and A. D. Chave, 2001: Low power instrumentation to measure barotropic fluctuations. Proc. IEEE Oceans 2001, Honolulu, HI, IEEE, 1886-1890.

Berdichevsky, M. N., L. L. Vanyan, and V. I. Dmitriev, 1989: Methods used in the USSR to reduce near-surface inhomogeneity effects on deep magnetotelluric sounding. Phys. Earth Planet. Inter., 53, 194-206.

Boström, R., 1964: A model of auroral electrojets. J. Geophys. Res., 69, 4883-4999.

Campbell, W. H., 1997: Introduction to Geomagnetic Fields. Cambridge University Press, 290 pp.

Chapman, S., 1935: The electric current systems of magnetic storms. Terr. Magn. Atmos. Electr., 40, 349-370.

Chave, A. D., and D. S. Luther, 1990: Low frequency, motionally induced electromagnetic fields in the ocean, 1. Theory. J. Geophys. Res., 95, 7185-7200.

_ , and J. T. Smith, 1994: On electric and magnetic field galvanic distortion tensor decompositions. J. Geophys. Res., 99, 46694682.

—_, and D. J. Thomson, 2003a: A bounded influence regression estimator based on the statistics of the hat matrix. J. Roy. Stat. Soc., 52C, 307-322.

- and - 2003b: Bounded influence estimation of magnetotelluric response functions. Geophys. J. Int., in press.

—_ A. H. Flosadottir, and C. S. Cox, 1990: Some comments on seabed propagation of ULF/ELF electromagnetic fields. Radio Sci., 25, 825-836.

Cox, C. S., 1980: Electromagnetic induction in the oceans and inferences on the constitution of the earth. Geophys. Surv., 4, 137156.

Evans, R. L., and Coauthors, 1999: Asymmetric mantle electrical structure beneath the East Pacific Rise at $17^{\circ} \mathrm{S}$. Science, 286, $752-756$.

Filloux, J. H., 1987: Instrumentation and experimental methods for oceanic studies. Geomagnetism, J. A. Jacobs, Ed., Vol. 1, Academic Press, 143-248.

Flosadottir, A. H., J. C. Larsen, and J. T. Smith, 1997a: Motional induction in North Atlantic circulation models. J. Geophys. Res., 102, $10353-10372$.

- —, and — 1997b: The relation of seafloor voltages to ocean transports in North Atlantic circulation models: Model results and practical considerations for transport modeling. $J$. Phys. Oceanogr., 27, 1547-1565.

Groom, R. W., and R. C. Bailey, 1989: Decomposition of magnetotelluric impedance tensors in the presence of local three-dimensional galvanic distortion. J. Geophys. Res., 94, 1913-1925. , and _ 1991: Analytic investigations of the effects of nearsurface three-dimensional galvanic scatterers on MT tensor decompositions. Geophysics, 56, 496-518.

_ , and K. Bahr, 1992: Corrections for near surface effects: Decomposition of the magnetotelluric impedance tensor and scaling 
corrections for regional resistivities: A tutorial. Surv. Geophys., 13, 341-380.

Habashy, T. M., R. W. Groom, and B. R. Spies, 1993: Beyond the Born and Rytov approximations: A nonlinear approach to electromagnetic scattering. J. Geophys. Res., 98, 1759-1775.

Hayes, D. E., and J. R. LaBrecque, 1991: Sediment isopachs: CircumAntarctic to $30^{\circ} \mathrm{S}$. Marine Geological and Geophysical Atlas of the Circum-Antarctic to $30^{\circ} \mathrm{S}$, D. E. Hayes, Ed., Antarctic Research Series, Vol. 54, Amer. Geophys. Union, 29-36.

Hughes, T. J., and G. Rostoker, 1979: A comprehensive model current system for high latitude magnetic activity, I. The steady state system. Geophys. J. Roy. Astron. Soc., 58, 525-569.

Jiracek, G. R., 1990: Near-surface and topographic distortions in electromagnetic induction. Surv. Geophys., 11, 163-203.

Jones, A. G., 1983: The problem of current channeling: A critical review. Surv. Geophys., 6, 79-122.

Kisabeth, J. L., and G. Rostoker, 1977: Modeling of three-dimensional current systems associated with magnetic substorms. Geophys. J. Roy. Astron. Soc., 49, 655-683.

Larsen, J. C., 1992: Transport and heat flux of the Florida Current at $27^{\circ} \mathrm{N}$ derived from cross-stream voltages and profiling data: Theory and observations. Philos. Trans. Roy. Soc. London, A338, 169-236.

Lizarralde, D., A. D. Chave, J. G. Hirth, and A. Schultz, 1995: Long period magnetotelluric study using Hawaii-to-California submarine cable data: Implications for mantle conductivity. J. Geophys. Res., 100, 17 837-17 854.

Luther, D. S., and A. D. Chave, 1993: Observing "integrating" variables in the ocean. Statistical Methods in Physical Oceanography: Proc. 'Aha Huliko'a Hawaiian Winter Workshop, Honolulu, HI, University of Hawaii at Manoa, 103-130.

_ J. H. Filloux, and A. D. Chave, 1991: Low-frequency, motionally induced electromagnetic fields in the ocean, 2, Electric field and Eulerian current comparison from BEMPEX. J. Geophys. Res., 96, 12 797-12 814.

- A. D. Chave, J. Church, J. H. Filloux, J. Richman, S. Rintoul, and D. R. Watts, 1997: The Sub-Antarctic Flux and Dynamics Experiment (SAFDE). International WOCE Newsletter, No. 29, WOCE International Project Office, Southampton, United Kingdom, 32-35.

Macmillan, S., D. R. Barraclough, J. M. Quinn, and R. J. Coleman, 1997: The 1995 revision of the joint US/UK geomagnetic field models, I. Secular variation. J. Geomagn. Geoelectr., 49, 229 243.

Mareschal, M., 1981: Source effects and the interpretation of geomagnetic sounding data at sub-auroral latitudes. Geophys. J. Roy. Astron. Soc., 67, 125-136.

_ 1986: Modeling of natural sources of magnetospheric origin in the interpretation of regional induction studies: A review. Surv. Geophys., 8, 261-300.

Meinen, C. S., and D. R. Watts, 1998: Calibrating inverted echo sounders equipped with pressure sensors. J. Atmos. Oceanic Technol., 15, 1339-1345.

- and - 2000: Vertical structure and transport on a transect across the North Atlantic Current near $42^{\circ} \mathrm{N}$ : Time series and mean. J. Geophys. Res., 105, 21 869-21 891.
- and D. S. Luther, 2002: Mooring motion when the pressure sensors fail: A method employing inverted echo sounders. $J$. Atmos. Oceanic Technol., 19, 1451-1460.

- D. R. Watts, K. L. Tracey, A. D. Chave, and J. G. Richman, 2002: Combining inverted echo sounder and horizontal electric field recorder measurements to obtain absolute velocity profiles. J. Atmos. Oceanic Technol., 19, 1653-1664.

Nishida, A., 1978: Geomagnetic Diagnosis of the Magnetosphere. Springer-Verlag, $256 \mathrm{pp}$.

Nolasco, R., P. Tarits, J. H. Filloux, and A. D. Chave, 1998: Magnetotelluric imaging of the Society Islands hot spot. J. Geophys. Res., 103, 30 287-30 310.

Petitt, R. A., J. H. Filloux, and A. D. Chave, 1992: Technology for the measurement of oceanic low frequency electric fields. Proc. IEEE Oceans '92, Vol. 2, Newport, RI, IEEE, 642-647.

Quinn, J. M., R. J. Coleman, S. Macmillan, and D. R. Barraclough, 1997: The 1995 revision of the joint US/UK geomagnetic field models, II. Main field. J. Geomagn. Geoelectr., 49, 245-261.

Rostoker, G., and T. J. Hughes, 1979: A comprehensive model current system for high-latitude magnetic activity, II: The substorm component. Geophys. J. Roy. Astron. Soc., 58, 571-581.

Sanford, T. B., 1971: Motionally-induced electric and magnetic fields in the sea. J. Geophys. Res., 76, 3476-3492.

— 1986: Recent improvements in ocean current measurement from motional electric fields and currents. Proc. IEEE Third Working Conf. on Current Measurement, Airlie, VA, IEEE, 65-76.

— , R. G. Drever, J. H. Dunlap, and W. E. Johns, 1995: Barotropic flows observed by the towed transport meter. Proc. IEEE Fifth Working Conf. on Current Measurement, St. Petersburg, FL, IEEE, 24-29.

Smith, W. H. F., and D. T. Sandwell, 1997: Global sea floor topography from satellite altimetry and ship depth soundings. Science, 277, $1956-1962$.

Spain, P., and T. B. Sanford, 1987: Accurately monitoring the Florida Current with motionally induced voltages. J. Mar. Res., 45, 843 870.

Stuart, A., and J. K. Ord, 1994: Distribution Theory. Vol. 1, Kendall's Advanced Theory of Statistics, Edward Arnold, 676 pp.

—,$\ldots$, and S. Arnold, 1999: Classical Inference and the Linear Model. Vol. 2A, Kendall's Advanced Theory of Statistics, Edward Arnold, 885 pp.

Thomson, D. J., and A. D. Chave, 1991: Jackknife error estimates for spectra, coherences, and transfer functions. Advances in Spectral Analysis and Array Processing, Vol. 1, S. Haykin, Ed., Prentice Hall, 58-113.

Tracey, K. L., S. D. Howden, and D. R. Watts, 1997: IES calibration and mapping procedures. J. Atmos. Oceanic Technol., 14, 14831493.

Tyler, R. H., L. A. Mysak, and J. M. Oberhuber, 1997a: Electromagnetic fields generated by a 3-D global ocean circulation. $J$. Geophys. Res., 102, 5531-5551.

_ _ T. B. Sanford, and J. M. Oberhuber, 1997b: Geophysical challenges in using large-scale ocean-generated EM fields to determine the ocean flow. J. Geomagn. Geoelectr., 49, 1351-1372.

Yaghijian, A. D., 1980: Electric dyadic Green's functions in the source region. Proc. IEEE, 68, 248-263. 